\title{
Some Properties of Intuitionistic Fuzzy Soft Rings
}

\author{
B. A. Ersoy, ${ }^{1}$ S. Onar, ${ }^{1}$ K. Hila, ${ }^{2}$ and B. Davvaz ${ }^{3}$ \\ ${ }^{1}$ Department of Mathematics, Yildiz Technical University, 81270 Istanbul, Turkey \\ ${ }^{2}$ Department of Mathematics \& Computer Science, Faculty of Natural Sciences, University of Gjirokastra, Albania \\ ${ }^{3}$ Department of Mathematics, Yazd University, Yazd, Iran
}

Correspondence should be addressed to B. Davvaz; davvaz@yazd.ac.ir

Received 8 November 2012; Revised 13 January 2013; Accepted 21 February 2013

Academic Editor: Jianming Zhan

Copyright (C) 2013 B. A. Ersoy et al. This is an open access article distributed under the Creative Commons Attribution License, which permits unrestricted use, distribution, and reproduction in any medium, provided the original work is properly cited.

Maji et al. introduced the concept of intuitionistic fuzzy soft sets, which is an extension of soft sets and intuitionistic fuzzy sets. In this
paper, we apply the concept of intuitionistic fuzzy soft sets to rings. The concept of intuitionistic fuzzy soft rings is introduced and
some basic properties of intuitionistic fuzzy soft rings are given. Intersection, union, AND, and OR operations of intuitionistic fuzzy
soft rings are defined. Then, the deffinitions of intuitionistic fuzzy soft ideals are proposed and some related results are considered.

\section{Introduction}

Uncertain or imprecise data are inherent and pervasive in many important applications in the areas such as economics, engineering, environment, social science, medical science, and business management. Uncertain data in those applications could be caused by data randomness, information incompleteness, limitations of measuring instrument, delayed data updates, and so forth. Due to the importance of those applications and the rapidly increasing amount of uncertain data collected and accumulated, research on effective and efficient techniques that are dedicated to modeling uncertain data and tackling uncertainties has attracted much interest in recent years and yet remained challenging at large. There have been a great amount of research and applications in the literature concerning some special tools like probability theory, (intuitionistic) fuzzy set theory, rough set theory, vague set theory, and interval mathematics. However, all of these have theirs advantages as well as inherent limitations in dealing with uncertainties. One major problem shared by those theories is their incompatibility with the parameterizations tools. Soft set theory [1] was firstly proposed by a Russian researcher, Molodtsov, in 1999 to overcome these difficulties.

At present, work on the extension of soft set theory is progressing rapidly. Maji et al. proposed the concept of fuzzy soft set [2] and then gave its application. Roy and Maji presented a method of object recognition from an imprecise multiobserver data [3]. Yao et al. proposed the concept of fuzzy sets and defined some operations on fuzzy soft sets [4].

From the above discussion, we can see that all of these works are based on Zadeh's fuzzy sets theory which was generalized to intuitionistic fuzzy sets by Atanassov [5]. The concept of intuitionistic fuzzy set was introduced and studied by Atanassov [5, 6] as a generalization of the notion of fuzzy set. Some applications of intuitionistic fuzzy sets are discussed in [7]. In [8-12], Davvaz et al. applied the concept of intuitionistic fuzzy sets to algebraic hyperstructures and some related properties are investigated.

The purpose of this paper is to deal with the algebraic structure of ring by applying intuitionistic fuzzy soft theory. We introduce the notion of intuitionistic fuzzy soft ring and study some of its characterization of operations and algebraic properties.

\section{Preliminaries}

In this section, for the sake of completeness, we first cite some useful definitions and results.

Definition 1 (see [13]). A fuzzy subset $\mu$ in a set $X$ is a function $\mu: X \rightarrow[0,1]$ 
Definition 2 (see [1]). Let $U$ be an initial universe and $E$ a set of parameters. Let $P(U)$ denote the power set of $U$ and $A \subset E$. A pair $(F, A)$ is called a soft set over $U$, where $F$ is a mapping given by $F: A \rightarrow P(U)$. In other words, a soft set over $U$ is a parameterized family of subsets of the universe $U$.

Definition 3 (see [14]). Let $U$ be an initial universe, and $E$ a set of parameters and $F S(U)$ denotes the fuzzy power set of $U$ and $A \subset E$. A pair $(F, A)$ is called a fuzzy soft set over $U$, where $F$ is a mapping given by $F: A \rightarrow F S(U)$. A fuzzy soft set is a parameterized family of fuzzy subsets of $U$.

Definition 4 (see [14]). An intuitionistic fuzzy set $A$ on the universe $X$ can be defined as follows:

$$
A=\left\{\left\langle x, \mu_{A}(x), v_{A}(x)\right\rangle \mid x \in X\right\},
$$

where $\mu_{A}(x): X \rightarrow[0,1]$ and $v_{A}(x): X \rightarrow[0,1]$ with the following property:

$$
0 \leq \mu_{A}(x)+v_{A}(x) \leq 1, \quad \forall x \in X
$$

The values $\mu_{A}(x)$ and $v_{A}(x)$ denote the degree of membership and nonmembership of $x$ to $A$, respectively.

Lemma 5 (see $[7,15])$. Let $A=\left\{\left\langle x, \mu_{A}(x), v_{A}(x)\right\rangle \mid x \in X\right\}$ be an intuitionistic fuzzy set in $X$ and let $r, t \in[0,1]$ such that $r+t \leq 1$. One defines

(1) $\square A=\left\{\left\langle x, \mu_{A}(x), \mu_{A}^{c}(x)\right\rangle \mid x \in X\right\}$,

(2) $\diamond A=\left\{\left\langle x, v_{A}^{c}(x), v_{A}(x)\right\rangle \mid x \in X\right\}$,

(3) $P_{r, t}(A)=\left\{\left(x, \max \left\{r, \mu_{A}(x)\right\}, \min \left\{t, v_{A}(x)\right\}\right) \mid x \in X\right\}$,

(4) $Q_{r, t}(A)=\left\{\left(x, \min \left\{r, \mu_{A}(x)\right\}, \max \left\{t, v_{A}(x)\right\}\right) \mid x \in X\right\}$.

Then, $\square A, \diamond A, P_{r, t}(A)$, and $Q_{r, t}(A)$ are intuitionistic fuzzy sets in $X$.

Definition 6 (see [16]). Let $U$ be an initial universe and $E$ a set of parameters and $\operatorname{IFS}(U)$ denotes the intuitionistic fuzzy power set of $U$ and $A \subset E$. A pair $(\widetilde{F}, A)$ is called an intuitionistic fuzzy soft set over $U$, where $F$ is a mapping given by $F: A \rightarrow \operatorname{IFS}(U)$.

An intuitionistic fuzzy soft set is a parameterized family of intuitionistic fuzzy subsets of $U$; a fuzzy soft set is a special case of an intuitionistic fuzzy soft set, because when all the intuitionistic fuzzy subset of $U$ degenerates into fuzzy subsets, the corresponding intuitionistic fuzzy soft set degenerates into a fuzzy soft set.

In general, for all $\varepsilon \in A \subseteq E, \widetilde{F}(\varepsilon)$ is an intuitionistic fuzzy set on $U$, which is called the intuitionistic fuzzy set of parameter $\varepsilon$. The intuitionistic value

$$
\left\langle\mu_{\widetilde{F}(\varepsilon)}(x), v_{\widetilde{F}(\varepsilon)}(x)\right\rangle
$$

denotes the degree that object $x \in U$ holds parameter $\varepsilon$. $\widetilde{F}(\varepsilon)$ can be written as following:

$$
\widetilde{F}(\varepsilon)=\left\{\left\langle x, \mu_{\widetilde{F}(\varepsilon)}(x), v_{\widetilde{F}(\varepsilon)}(x)\right\rangle \mid x \in U\right\} .
$$

If for all $x \in U, \mu_{\widetilde{F}(\varepsilon)}(x)+v_{\widetilde{F}(\varepsilon)}(x)=1$, then $\widetilde{F}(\varepsilon)$ degenerates into a fuzzy set; if for all $x \in U$ and for all $\varepsilon \in A \subseteq E$, $\mu_{\widetilde{F}(\varepsilon)}(x)+v_{\widetilde{F}(\varepsilon)}(x)=1$, then the intuitionistic fuzzy soft set $(\widetilde{F}, A)$ degenerates into a fuzzy soft set.

Example 7. Let $(\widetilde{F}, A)$ describe the character of the students with respect to the given parameter, for finding the best student of an academic year. Let the set of students under consideration be $U=\left\{s_{1}, s_{2}, s_{3}, s_{4}\right\}$. Let $A \subseteq E$ and

$$
\begin{aligned}
A=\{r & =\text { "result", } c=\text { "conduct", } \\
& \quad g=\text { "games and sports performances" }\} .
\end{aligned}
$$

Let

$$
\begin{aligned}
& \widetilde{F}(r)=\left\{\left(s_{1}, 0.8,0.1\right),\left(s_{2}, 0.9,0.05\right),\right. \\
&\left.\left(s_{3}, 0.85,0.1\right),\left(s_{4}, 0.75,0.2\right)\right\}, \\
& \widetilde{F}(c)=\left\{\left(s_{1}, 0.6,0.3\right),\left(s_{2}, 0.65,0.2\right),\right. \\
&\left.\left(s_{3}, 0.7,0.2\right),\left(s_{4}, 0.65,0.2\right)\right\}, \\
& \widetilde{F}(g)=\left\{\left(s_{1}, 0.75,0.2\right),\left(s_{2}, 0.5,0.3\right),\right. \\
&\left.\left(s_{3}, 0.5,0.4\right),\left(s_{4}, 0.7,0.2\right)\right\}
\end{aligned}
$$

Then, the family $\{\widetilde{F}(r), \widetilde{F}(c), \widetilde{F}(g)\}$ of $\operatorname{IFS}(U)$ is an intuitionistic fuzzy soft set.

Definition 8 (see $[16])$. Let $(\widetilde{F}, A)$ and $(\widetilde{G}, B)$ be two intuitionistic fuzzy soft sets over $U$. Then, $(\widetilde{F}, A)$ is said to be an intuitionistic fuzzy soft subset of $(\widetilde{G}, B)$ if

(1) $A \subset B$,

(2) $\widetilde{F}(\varepsilon)$ is an intuitionistic fuzzy subset of $\widetilde{G}(\varepsilon)$, for all $\varepsilon \in$ A.

We denote the above inclusion relationship by $(\widetilde{F}, A) \widetilde{\subseteq}(\widetilde{G}, B)$. Similarly, $(\widetilde{F}, A)$ is called an intuitionistic fuzzy soft superset of $(\widetilde{G}, B)$ if $(\widetilde{G}, B)$ is an intuitionistic fuzzy soft subset of $(\widetilde{F}, A)$. We denoted the above relationship by $(\widetilde{F}, A) \widetilde{\mathcal{J}}(\widetilde{G}, B) \cdot(\widetilde{F}, A)$ and $(\widetilde{G}, B)$ over a universe $U$ are said to be intuitionistic fuzzy soft equals if $(\widetilde{F}, A) \widetilde{\subseteq}(\widetilde{G}, B)$ and $(\widetilde{G}, B) \widetilde{\subseteq}(\widetilde{F}, A)$.

Definition 9 (see $[16])$. Let $(\widetilde{F}, A)$ and $(\widetilde{G}, B)$ be two intuitionistic fuzzy soft sets over a universe $U$. Then, "( $\widetilde{F}, A)$ and $(\widetilde{G}, B)$ " is denoted by $(\widetilde{F}, A) \widetilde{\wedge}(\widetilde{G}, B)$ and is defined by $(\widetilde{F}, A) \widetilde{\wedge}(\widetilde{G}, B)=(\widetilde{H}, A \times B)$, where $\widetilde{H}(\alpha, \beta)=\widetilde{F}(\alpha) \cap \widetilde{G}(\beta)$, for all $(\alpha, \beta) \in A \times B$.

Definition 10 (see [16]). Let $(\widetilde{F}, A)$ and $(\widetilde{G}, B)$ be two intuitionistic fuzzy soft sets over a universe $U$. Then, "( $\widetilde{F}, A)$ or $(\widetilde{G}, B)$ " is denoted by $(\widetilde{F}, A) \widetilde{V}(\widetilde{G}, B)$ and is defined by $(\widetilde{F}, A) \widetilde{V}(\widetilde{G}, B)=(\widetilde{H}, A \times B)$, where $\widetilde{H}(\alpha, \beta)=\widetilde{F}(\alpha) \cup \widetilde{G}(\beta)$, for all $(\alpha, \beta) \in A \times B$. 
Definition 11 (see [16]). The intersection of two intuitionistic fuzzy soft sets $(\widetilde{F}, A)$ and $(\widetilde{G}, B)$ over a universe $U$ is an intuitionistic fuzzy soft set denoted by $(\widetilde{H}, C)$, where $C=A \cup B$ and

$$
\widetilde{H}(\varepsilon)= \begin{cases}\widetilde{F}(\varepsilon) & \text { if } \varepsilon \in A-B, \\ \widetilde{G}(\varepsilon) & \text { if } \varepsilon \in B-A, \\ \min \{\widetilde{F}(\varepsilon), \widetilde{G}(\varepsilon)\} & \text { if } \varepsilon \in A \cap B,\end{cases}
$$

for all $\varepsilon \in C$. This is denoted by $(\widetilde{H}, C)=(\widetilde{F}, A) \widetilde{\cap}(\widetilde{G}, B)$.

Definition 12 (see [16]). The union of two intuitionistic fuzzy soft sets $(\widetilde{F}, A)$ and $(\widetilde{G}, B)$ over a universe $U$ is an intuitionistic fuzzy soft set denoted by $(\widetilde{H}, C)$, where $C=A \cup B$ and

$$
\widetilde{H}(\varepsilon)= \begin{cases}\widetilde{F}(\varepsilon) & \text { if } \varepsilon \in A-B, \\ \widetilde{G}(\varepsilon) & \text { if } \varepsilon \in B-A, \\ \max \{\widetilde{F}(\varepsilon), \widetilde{G}(\varepsilon)\} & \text { if } \varepsilon \in A \cap B,\end{cases}
$$

for all $\varepsilon \in C$. This is denoted by $(\widetilde{H}, C)=(\widetilde{F}, A) \widetilde{U}(\widetilde{G}, B)$.

In contrast with the above definitions of union and intersection of intuitionistic fuzzy soft sets, we may sometimes adopt different definitions of union and intersection given as follows.

Definition 13 (see $[16])$. Let $(\widetilde{F}, A)$ and $(\widetilde{G}, B)$ be two intuitionistic fuzzy soft sets over a universe $U$ such that $A \cap B \neq \emptyset$. The biunion of $(\widetilde{F}, A)$ and $(\widetilde{G}, B)$ is defined to be the intuitionistic fuzzy soft set $(\widetilde{H}, C)$, where $C=A \cap B$ and $\widetilde{H}(\varepsilon)=\widetilde{F}(\varepsilon) \cup \widetilde{G}(\varepsilon)$ for all $\varepsilon \in C$. This is denoted by $(\widetilde{H}, C)=(\widetilde{F}, A) \widetilde{\sqcup}(\widetilde{G}, B)$.

Definition 14 (see $[16])$. Let $(\widetilde{F}, A)$ and $(\widetilde{G}, B)$ be two intuitionistic fuzzy soft sets over a universe $U$ such that $A \cap B \neq \emptyset$. The bi-intersection $(\widetilde{F}, A)$ and $(\widetilde{G}, B)$ is defined to be the intuitionistic fuzzy soft set $(\widetilde{H}, C)$, where $C=A \cap B$ and $\widetilde{H}(\varepsilon)=\widetilde{F}(\varepsilon) \cap \widetilde{G}(\varepsilon)$ for all $\varepsilon \in C$. This is denoted by $(\widetilde{H}, C)=$ $(\widetilde{F}, A) \widetilde{\Pi}(\widetilde{G}, B)$.

Definition 15. A fuzzy set $\mu$ in $\operatorname{ring} R$ is called a fuzzy ideal of $R$, if for all $x, y \in R$ the following conditions hold:
(1) $\mu(x-y) \geq \min \{\mu(x), \mu(y)\}$,
(2) $\mu(x y) \geq \max \{\mu(x), \mu(y)\}$.

\section{Intuitionistic Fuzzy Soft Rings}

Definition 16. A pair $(\widetilde{F}, A)$ is called an intuitionistic fuzzy soft ring over $R$, where $\widetilde{F}$ is a mapping given by

$$
\begin{gathered}
\widetilde{F}: A \longrightarrow([0,1] \times[0,1])^{R}, \quad \widetilde{F}(\varepsilon): R \longrightarrow[0,1] \times[0,1], \\
\widetilde{F}(\varepsilon)=\left\{\left(x, \mu_{\widetilde{F}(\varepsilon)}(x), v_{\widetilde{F}(\varepsilon)}(x)\right): x \in R\right\} \quad \forall \varepsilon \in A,
\end{gathered}
$$

if for all $x, y \in R$ the following conditions hold:

$$
\begin{aligned}
& \text { (1) } \mu_{\widetilde{F}(\varepsilon)}(x-y) \geq \mu_{\widetilde{F}(\varepsilon)}(x) \wedge \mu_{\widetilde{F}(\varepsilon)}(y) \text { and } v_{\widetilde{F}(\varepsilon)}(x-y) \leq \\
& v_{\widetilde{F}(\varepsilon)}(x) \vee v_{\widetilde{F}(\varepsilon)}(y), \\
& \text { (2) } \mu_{\widetilde{F}(\varepsilon)}(x y) \geq \mu_{\widetilde{F}(\varepsilon)}(x) \wedge \mu_{\widetilde{F}(\varepsilon)}(y) \text { and } v_{\widetilde{F}(\varepsilon)}(x y) \leq \\
& v_{\widetilde{F}(\varepsilon)}(x) \vee v_{\widetilde{F}(\varepsilon)}(y) .
\end{aligned}
$$

Definition 17. For two intuitionistic fuzzy soft $\operatorname{rings}(\widetilde{F}, A)$ and $(\widetilde{G}, B)$ over a universe $R$, we say that $(\widetilde{F}, A)$ is an intuitionistic fuzzy soft subring of $(\widetilde{G}, B)$ and write $(\widetilde{F}, A) \subseteq(\widetilde{G}, B)$ if

(i) $A \subseteq B$,

(ii) for each $x \in R, \varepsilon \in A, \mu_{\widetilde{F}(\varepsilon)}(x) \leq \mu_{\widetilde{G}(\varepsilon)}(x)$ and $v_{\widetilde{F}(\varepsilon)}(x) \geq v_{\widetilde{G}(\varepsilon)}(x)$.

Definition 18. Two intuitionistic fuzzy soft rings $(\widetilde{F}, A)$ and $(\widetilde{G}, B)$ over a universe $R$ are said to be equal if $(\widetilde{F}, A) \subseteq(\widetilde{G}, B)$ and $(\widetilde{G}, B) \subseteq(\widetilde{F}, A)$.

Theorem 19. Let $(\widetilde{F}, A)$ and $(\widetilde{G}, B)$ be two intuitionistic fuzzy soft rings over a universe $R$. If $\widetilde{F}(\varepsilon) \leq \widetilde{G}(\varepsilon)$ for all $\varepsilon \in A$, then $(\widetilde{F}, A)$ is an intuitionistic fuzzy soft subring of $(\widetilde{G}, B)$.

Proof. The proof is straightforward.

Definition 20. The union of two intuitionistic fuzzy soft rings $(\widetilde{F}, A)$ and $(\widetilde{G}, B)$ over a universe $R$ is denoted by $(\widetilde{F}, A) \widetilde{U}$ $(\widetilde{G}, B)$ and is defined by an intuitionistic fuzzy soft $\operatorname{ring} \widetilde{H}$ : $A \cup B \rightarrow[0,1]^{R}$ such that for each $\varepsilon \in A \cup B$,

$$
\widetilde{H}(\varepsilon)=\left\{\begin{aligned}
\left\langle x, \mu_{\widetilde{F}(\varepsilon)}(x), v_{\widetilde{F}(\varepsilon)}(x)\right\rangle & \\
& \text { if } \varepsilon \in A-B \\
\left\langle x, \mu_{\widetilde{G}(\varepsilon)}(x), v_{\widetilde{G}(\varepsilon)}(x)\right\rangle & \\
& \text { if } \varepsilon \in B-A \\
\left\langle x, \mu_{\widetilde{F}(\varepsilon)}(x) \vee \mu_{\widetilde{G}(\varepsilon)}(x), v_{\widetilde{F}(\varepsilon)}(x) \wedge v_{\widetilde{G}(\varepsilon)}(x)\right\rangle & \text { if } \varepsilon \in A \cap B .
\end{aligned}\right.
$$

This is denoted by $(\widetilde{H}, C)=(\widetilde{F}, A) \widetilde{\cup}(\widetilde{G}, B)$, where $C=$ $A \cup B$.

Theorem 21. If $(\widetilde{F}, A)$ and $(\widetilde{G}, B)$ are two intuitionistic fuzzy soft rings over a universe $R$, then, so are $(\widetilde{F}, A) \widetilde{\cup}(\widetilde{G}, B)$.

Proof. For any $\varepsilon=(\gamma, \beta) \in A \cup B$ and $x, y \in R$, we consider the following cases.

Case 1. Let $\varepsilon \in A-B$. Then,

$$
\begin{aligned}
\mu_{\widetilde{H}(\varepsilon)}(x-y) & =\mu_{\widetilde{F}(\varepsilon)}(x-y) \\
& \geq \mu_{\widetilde{F}(\varepsilon)}(x) \wedge \mu_{\widetilde{F}(\varepsilon)}(y) \\
& =\mu_{\widetilde{H}(\varepsilon)}(x) \wedge \mu_{\widetilde{H}(\varepsilon)}(y),
\end{aligned}
$$




$$
\begin{aligned}
\mu_{\widetilde{H}(\varepsilon)}(x y)= & \mu_{\widetilde{F}(\varepsilon)}(x y) \\
\geq & \mu_{\widetilde{F}(\varepsilon)}(x) \wedge \mu_{\widetilde{F}(\varepsilon)}(y) \\
= & \mu_{\widetilde{H}(\varepsilon)}(x) \wedge \mu_{\widetilde{H}(\varepsilon)}(y), \\
v_{\widetilde{H}(\varepsilon)}(x-y) & =v_{\widetilde{F}(\varepsilon)}(x-y) \\
& \leq v_{\widetilde{F}(\varepsilon)}(x) \vee v_{\widetilde{F}(\varepsilon)}(y) \\
& =v_{\widetilde{H}(\varepsilon)}(x) \vee v_{\widetilde{H}(\varepsilon)}(y), \\
v_{\widetilde{H}(\varepsilon)}(x y)= & v_{\widetilde{F}(\varepsilon)}(x y) \\
\leq & v_{\widetilde{F}(\varepsilon)}(x) \vee v_{\widetilde{F}(\varepsilon)}(y) \\
= & v_{\widetilde{H}(\varepsilon)}(x) \vee v_{\widetilde{H}(\varepsilon)}(y) .
\end{aligned}
$$

Case 2. Let $\varepsilon \in B-A$. Then, analogous to the proof of Case 1 , we have

$$
\begin{aligned}
& \mu_{\widetilde{H}(\varepsilon)}(x-y) \geq \mu_{\widetilde{H}(\varepsilon)}(x) \wedge \mu_{\widetilde{H}(\varepsilon)}(y), \\
& \mu_{\widetilde{H}(\varepsilon)}(x \alpha y) \geq \mu_{\widetilde{H}(\varepsilon)}(x) \wedge \mu_{\widetilde{H}(\varepsilon)}(y), \\
& v_{\widetilde{H}(\varepsilon)}(x-y) \leq v_{\widetilde{H}(\varepsilon)}(x) \vee v_{\widetilde{H}(\varepsilon)}(y), \\
& v_{\widetilde{H}(\varepsilon)}(x y) \leq v_{\widetilde{H}(\varepsilon)}(x) \vee v_{\widetilde{H}(\varepsilon)}(y) .
\end{aligned}
$$

Case 3. Let $\varepsilon \in A \cap B$. In this case the proof is straightforward. Thus, in any cases, we have

$$
\begin{aligned}
& \mu_{\widetilde{H}(\varepsilon)}(x-y) \geq \mu_{\widetilde{H}(\varepsilon)}(x) \wedge \mu_{\widetilde{H}(\varepsilon)}(y), \\
& \mu_{\widetilde{H}(\varepsilon)}(x y) \geq \mu_{\widetilde{H}(\varepsilon)}(x) \wedge \mu_{\widetilde{H}(\varepsilon)}(y), \\
& v_{\widetilde{H}(\varepsilon)}(x-y) \leq v_{\widetilde{H}(\varepsilon)}(x) \vee v_{\widetilde{H}(\varepsilon)}(y), \\
& v_{\widetilde{H}(\varepsilon)}(x y) \leq v_{\widetilde{H}(\varepsilon)}(x) \vee v_{\widetilde{H}(\varepsilon)}(y) .
\end{aligned}
$$

Therefore, $(\widetilde{F}, A) \widetilde{\cup}(\widetilde{G}, B)$ is an intuitionistic fuzzy soft ring.

Definition 22. The intersection of two intuitionistic fuzzy soft rings $(\widetilde{F}, A)$ and $(\widetilde{G}, B)$ over a universe $R$ is denoted by $(\widetilde{F}, A) \widetilde{\cap}$ $(\widetilde{G}, B)$ and is defined by

$$
\widetilde{H}(\varepsilon)=\left\{\begin{aligned}
\left\langle x, \mu_{\widetilde{F}(\varepsilon)}(x), v_{\widetilde{F}(\varepsilon)}(x)\right\rangle & \\
& \text { if } \varepsilon \in A-B \\
\left\langle x, \mu_{\widetilde{G}(\varepsilon)}(x), v_{\widetilde{G}(\varepsilon)}(x)\right\rangle & \\
& \text { if } \varepsilon \in B-A \\
\left\langle\mu_{\widetilde{F}(\varepsilon)}(x) \wedge \mu_{\widetilde{G}(\varepsilon)}(x), v_{\widetilde{F}(\varepsilon)}(x) \vee v_{\widetilde{G}(\varepsilon)}(x)\right\rangle & \text { if } \varepsilon \in A \cap B .
\end{aligned}\right.
$$

Theorem 23. If $(\widetilde{F}, A)$ and $(\widetilde{G}, B)$ are two intuitionistic fuzzy soft rings over a universe $R$, then, so are $(\widetilde{F}, A) \widetilde{\cap}(\widetilde{G}, B)$.

Proof. The proof is straightforward.

Definition 24. Let $(\widetilde{F}, A)$ and $(\widetilde{G}, B)$ be two intuitionistic fuzzy soft rings over a universe $R$. Then, " $(\widetilde{F}, A) \operatorname{AND}(\widetilde{G}, B)$ " is denoted by $(\widetilde{F}, A) \widetilde{\wedge}(\widetilde{G}, B)$ and is defined by $(\widetilde{F}, A) \widetilde{\wedge}(\widetilde{G}, B)=$ $(\widetilde{H}, C)$, where $C=A \times B$ and $\widetilde{H}: C \rightarrow([0,1] \times[0,1])^{R}$ is defined as

$$
\widetilde{H}(\alpha, \beta)=\widetilde{F}(\alpha) \widetilde{\cap} \widetilde{G}(\beta), \quad \forall(\alpha, \beta) \in C .
$$

Theorem 25. If $(\widetilde{F}, A)$ and $(\widetilde{G}, B)$ are two intuitionistic fuzzy soft rings over a universe $R$, then, so are $(\widetilde{F}, A) \widetilde{\wedge}(\widetilde{G}, B)$ and $(\widetilde{F}, A) \widetilde{\Pi}(\widetilde{G}, B)$.

Proof. For all $x, y \in R$ and $(\alpha, \beta) \in A \times B$ we have

$$
\begin{aligned}
\mu_{\widetilde{H}(\alpha, \beta)}(x-y)= & \left(\mu_{\widetilde{F}(\alpha)}(x-y) \cap \mu_{\widetilde{G}(\beta)}(x-y)\right) \\
\geq & \left(\mu_{\widetilde{F}(\alpha)}(x) \wedge \mu_{\widetilde{F}(\alpha)}(y)\right) \\
& \cap\left(\mu_{\widetilde{G}(\beta)}(x) \wedge \mu_{\widetilde{G}(\beta)}(y)\right) \\
= & \left(\mu_{\widetilde{F}(\alpha)}(x) \cap \mu_{\widetilde{G}(\beta)}(x)\right) \\
& \wedge\left(\mu_{\widetilde{F}(\alpha)}(y) \cap \mu_{\widetilde{G}(\beta)}(y)\right) \\
= & \mu_{\widetilde{H}(\alpha, \beta)}(x) \wedge \mu_{\widetilde{H}(\alpha, \beta)}(y), \\
\mu_{\widetilde{H}(\alpha, \beta)}(x y)= & \left(\mu_{\widetilde{F}(\alpha)}(x y) \cap \mu_{\widetilde{G}(\beta)}(x y)\right) \\
\geq & \left(\mu_{\widetilde{F}(\alpha)}(x) \wedge \mu_{\widetilde{F}(\alpha)}(y)\right) \\
& \cap\left(\mu_{\widetilde{G}(\beta)}(x) \wedge \mu_{\widetilde{G}(\beta)}(y)\right) \\
= & \left(\mu_{\widetilde{F}(\alpha)}(x) \cap \mu_{\widetilde{G}(\beta)}(x)\right) \\
& \wedge\left(\mu_{\widetilde{F}(\alpha)}(y) \cap \mu_{\widetilde{G}(\beta)}(y)\right) \\
= & \mu_{\widetilde{H}(\alpha, \beta)}(x) \wedge \mu_{\widetilde{H}(\alpha, \beta)}(y) .
\end{aligned}
$$

In a similar way, we have

$$
\begin{gathered}
v_{\widetilde{H}(\alpha, \beta)}(x-y) \leq v_{\widetilde{H}(\alpha, \beta)}(x) \vee v_{\widetilde{H}(\alpha, \beta)}(y), \\
v_{\widetilde{H}(\alpha, \beta)}(x y) \leq v_{\widetilde{H}(\alpha, \beta)}(x) \vee v_{\widetilde{H}(\alpha, \beta)}(y) .
\end{gathered}
$$

This is denoted by $(\widetilde{H}, C)=(\widetilde{F}, A) \widetilde{\cap}(\widetilde{G}, B)$, where $C=A \cup B$.

The case for $(\widetilde{F}, A) \widetilde{\Pi}(\widetilde{G}, B)$ can be similarly proved. 
Example 26 (see [17]). Consider the ring $R=\left\{h_{1}, h_{2}, h_{3}, h_{4}\right\}$ with the following tables:

$$
\begin{array}{c|llll}
+ & h_{1} & h_{2} & h_{3} & h_{4} \\
\hline h_{1} & h_{1} & h_{2} & h_{3} & h_{4} \\
h_{2} & h_{2} & h_{3} & h_{4} & h_{1} \\
h_{3} & h_{3} & h_{4} & h_{1} & h_{2} \\
h_{4} & h_{4} & h_{1} & h_{2} & h_{3} \\
\bullet & h_{1} & h_{2} & h_{3} & h_{4} \\
\hline h_{1} & h_{1} & h_{1} & h_{1} & h_{1} \\
h_{2} & h_{1} & h_{2} & h_{3} & h_{4} \\
h_{3} & h_{1} & h_{3} & h_{1} & h_{3} \\
h_{4} & h_{1} & h_{4} & h_{3} & h_{2}
\end{array}
$$

Let $A=\left\{e_{1}, e_{2}, e_{3}\right\}$ and $\widetilde{F}: A \rightarrow \mathscr{F}(R)$ be a set-valued function defined by

$$
\begin{aligned}
& \widetilde{F}\left(e_{1}\right)=\left\{\left(h_{1}, 0.84,0.11\right),\left(h_{2}, 0.56,0.31\right),\right. \\
&\left.\left(h_{3}, 0.83,0.12\right),\left(h_{4}, 0.69,0.15\right)\right\}, \\
& \widetilde{F}\left(e_{2}\right)=\left\{\left(h_{1}, 0.76,0.21\right),\left(h_{2}, 0.62,0.24\right),\right.\left.\left(h_{3}, 0.90,0.05\right),\left(h_{4}, 0.75,0.20\right)\right\}, \\
& \widetilde{F}\left(e_{3}\right)=\left\{\left(h_{1}, 0.94,0.04\right),\left(h_{2}, 0.74,0.17\right),\right. \\
&\left.\left(h_{3}, 0.68,0.26\right),\left(h_{4}, 0.86,0.13\right)\right\} .
\end{aligned}
$$

Obviously, $(\widetilde{F}, A)$ is an intuitionistic fuzzy soft set over $R$. Moreover, we see that $\left\langle\mu_{\widetilde{F}(\varepsilon)}(x), v_{\widetilde{F}(\varepsilon)}(x)\right\rangle$ is an intuitionistic fuzzy subring of $R$, for all $x \in A$. Therefore, $(\widetilde{F}, A)$ is an intuitionistic fuzzy soft ring over $R$.

Definition 27. Let $(\widetilde{F}, A)$ and $(\widetilde{G}, B)$ be two intuitionistic fuzzy soft rings over a universe $R$. Then, " $(\widetilde{F}, A)$ OR $(\widetilde{G}, B)$ " is denoted by $(\widetilde{F}, A) \widetilde{V}(\widetilde{G}, B)$ and is defined by $(\widetilde{F}, A) \widetilde{V}(\widetilde{G}, B)=$ $(\widetilde{H}, C)$, where $C=A \times B$ and $\widetilde{H}: C \rightarrow([0,1] \times[0,1])^{R}$ is defined as

$$
\widetilde{H}(\alpha, \beta)=\widetilde{F}(\alpha) \widetilde{U} \widetilde{G}(\beta), \quad \forall(\alpha, \beta) \in C .
$$

Theorem 28. If $(\widetilde{F}, A)$ and $(\widetilde{G}, B)$ are two intuitionistic fuzzy soft rings over a universe $R$, then, so are $(\widetilde{F}, A) \widetilde{V}(\widetilde{G}, B)$ and $(\widetilde{F}, A) \widetilde{\sqcup}(\widetilde{G}, B)$.

Proof. The proof is straightforward. results.

The following theorem is a generalization of previous

Theorem 29. Let $(\widetilde{F}, A)$ be an intuitionistic fuzzy soft rings over a universe $R$, and let $\left\{\left(\widetilde{F}_{i}, A_{i}\right)\right\}_{i \in I}$ be a nonempty family of intuitionistic fuzzy soft rings. Then, one has the following.

(1) $\widetilde{\Pi}_{i \in I}\left(\widetilde{F}_{i}, A_{i}\right)$ is an intuitionistic fuzzy soft ring over $R$.

(2) $\widetilde{\Lambda}_{i \in I}\left(\widetilde{F}_{i}, A_{i}\right)$ is an intuitionistic fuzzy soft ring over $R$.

(3) If $A_{i} \cap A_{j}=\emptyset$, for all $i, j \in I$, then $\widetilde{\mathrm{V}}_{i \in I}\left(\widetilde{F}_{i}, A_{i}\right)$ is an intuitionistic fuzzy soft ring over $R$.
Theorem 30. Let $N=(\widetilde{F}, A)$ be an intuitionistic fuzzy soft ring over a universe $R$, and let $r, t \in[0,1]$ such that $r+$ $t \leq 1$. Then the following are intuitionistic fuzzy soft rings: $(\square \widetilde{F}, A),(\diamond \widetilde{F}, A),\left(P_{r, t}(\widetilde{F}), A\right),\left(Q_{r, t}(\widetilde{F}), A\right)$ over $R$, where

$$
\begin{gathered}
(\square \widetilde{F}, A)=\square(\widetilde{F}, A)=\square N=\left(\mu_{\widetilde{F}(\varepsilon)}(x), \mu_{\widetilde{F}(\varepsilon)}^{c}(x), A\right), \\
(\diamond \widetilde{F}, A)=\diamond(\widetilde{F}, A)=\diamond N=\left(v_{\widetilde{F}(\varepsilon)}(x), v_{\widetilde{F}(\varepsilon)}^{c}(x), A\right), \\
\left(P_{r, t}(\widetilde{F}), A\right)=P_{r, t}(\widetilde{F}, A), \quad\left(Q_{r, t}(\widetilde{F}), A\right)=Q_{r, t}(\widetilde{F}, A) .
\end{gathered}
$$

Proof. Suppose that $(\widetilde{F}, A)$ is an intuitionistic fuzzy soft ring over $R$. Then,

$$
(\square \widetilde{F}, A)=\left(\mu_{\widetilde{F}(\varepsilon)}(x), \mu_{\widetilde{F}(\varepsilon)}^{c}(x), A\right) .
$$

For all $x, y \in R$,

$$
\begin{aligned}
\mu_{\widetilde{F}(\varepsilon)}(x-y) & \geq \mu_{\widetilde{F}(\varepsilon)}(x) \wedge \mu_{\widetilde{F}(\varepsilon)}(y), \\
\mu_{\widetilde{F}(\varepsilon)}(x y) & \geq \mu_{\widetilde{F}(\varepsilon)}(x) \wedge \mu_{\widetilde{F}(\varepsilon)}(y), \\
\mu_{\widetilde{F}(\varepsilon)}^{c}(x-y) & =1-\mu_{\widetilde{F}(\varepsilon)}(x-y) \\
& \leq\left(1-\mu_{\widetilde{F}(\varepsilon)}(x)\right) \wedge\left(1-\mu_{\widetilde{F}(\varepsilon)}(y)\right) \\
& =\mu_{\widetilde{F}(\varepsilon)}^{c}(x) \wedge \mu_{\widetilde{F}(\varepsilon)}^{c}(y), \\
\mu_{\widetilde{F}(\varepsilon)}^{c}(x y) & =1-\mu_{\widetilde{F}(\varepsilon)}(x y) \\
& \leq\left(1-\mu_{\widetilde{F}(\varepsilon)}(x)\right) \wedge\left(1-\mu_{\widetilde{F}(\varepsilon)}(y)\right) \\
& =\mu_{\widetilde{F}(\varepsilon)}^{c}(x) \wedge \mu_{\widetilde{F}(\varepsilon)}^{c}(y) .
\end{aligned}
$$

The proof of other cases is similar.

Definition 31. Let $(\widetilde{F}, A)$ and $(\widetilde{G}, B)$ be two intuitionistic fuzzy soft rings over a universe $R$. Then, the product of $(\widetilde{F}, A)$ and $(\widetilde{G}, B)$ is defined to be the intuitionistic fuzzy soft $\operatorname{ring}(\widetilde{F}$ 。 $\widetilde{G}, C)$, where $C=A \cup B$ and

$$
\mu_{\widetilde{F} \circ \widetilde{G}(\varepsilon)}(x)= \begin{cases}\mu_{\widetilde{F}(\varepsilon)}(x) & \text { if } \varepsilon \in A-B \\ \mu_{\widetilde{G}(\varepsilon)}(x) & \text { if } \varepsilon \in B-A \\ \bigvee_{x=a b}\left\{\mu_{\widetilde{F}(\varepsilon)}(a) \wedge \mu_{\widetilde{G}(\varepsilon)}(b)\right\} & \text { if } \varepsilon \in A \cap B,\end{cases}
$$

$$
v_{\widetilde{F} \circ \widetilde{G}(\varepsilon)}(x)= \begin{cases}v_{\widetilde{F}(\varepsilon)}(x) & \text { if } \varepsilon \in A-B \\ v_{\widetilde{G}(\varepsilon)}(x) & \text { if } \varepsilon \in B-A \\ \bigwedge_{x=a b}\left\{v_{\widetilde{F}(\varepsilon)}(a) \vee v_{\widetilde{G}(\varepsilon)}(b)\right\} & \text { if } \varepsilon \in A \cap B,\end{cases}
$$

for all $\varepsilon \in C$ and $x \in R$. This is denoted by $(\widetilde{F} \circ \widetilde{G}, C)=$ $(\widetilde{F}, A) \circ(\widetilde{G}, B)$. 
Theorem 32. If $(\widetilde{F}, A)$ and $(\widetilde{G}, B)$ are intuitionistic fuzzy soft rings over a universe $R$, then so is $(\widetilde{F}, A) \circ(\widetilde{G}, B)$.

Proof. Let $(\widetilde{F}, A)$ and $(\widetilde{G}, B)$ be two intuitionistic fuzzy soft rings over $R$. Then, for any $\varepsilon \in A \cup B$ and $x, y \in R$, we consider the following cases.

Case 1 . Let $\varepsilon \in A-B$. Then,

$$
\begin{aligned}
\mu_{\widetilde{F} \circ \widetilde{G}(\varepsilon)}(x-y) & =\mu_{\widetilde{F}(\varepsilon)}(x-y) \\
& \geq \mu_{\widetilde{F}(\varepsilon)}(x) \wedge \mu_{\widetilde{F}(\varepsilon)}(y) \\
& =\mu_{\widetilde{F} \circ \widetilde{G}(\varepsilon)}(x) \wedge \mu_{\widetilde{F} \circ \widetilde{G}(\varepsilon)}(y), \\
\mu_{\widetilde{F} \circ \widetilde{G}(\varepsilon)}(x y) & =\mu_{\widetilde{F}(\varepsilon)}(x y) \\
& \geq \mu_{\widetilde{F}(\varepsilon)}(x) \wedge \mu_{\widetilde{F}(\varepsilon)}(y) \\
& =\mu_{\widetilde{F} \circ \widetilde{G}(\varepsilon)}(x) \wedge \mu_{\widetilde{F} \circ \widetilde{G}(\varepsilon)}(y), \\
v_{\widetilde{F} \circ \widetilde{G}(\varepsilon)}(x-y) & =v_{\widetilde{F}(\varepsilon)}(x-y) \\
& \leq v_{\widetilde{F}(\varepsilon)}(x) \vee v_{\widetilde{F}(\varepsilon)}(y) \\
& =v_{\widetilde{F} \circ \widetilde{G}(\varepsilon)}(x) \vee v_{\widetilde{F} \circ \widetilde{G}(\varepsilon)}(y), \\
v_{\widetilde{F} \circ \widetilde{G}(\varepsilon)}(x y) & =v_{\widetilde{F}(\varepsilon)}(x y) \\
& \leq v_{\widetilde{F}(\varepsilon)}(x) \vee v_{\widetilde{F}(\varepsilon)}(y) \\
& =v_{\widetilde{F} \circ \widetilde{G}(\varepsilon)}(x) \vee v_{\widetilde{F} \circ \widetilde{G}(\varepsilon)}(y) .
\end{aligned}
$$

Case 2. Let $\varepsilon \in B-A$. Then, analogous to the proof of Case 1 , the proof is straightforward.

Case 3. Let $\varepsilon \in A \cap B$. Then,

$$
\begin{aligned}
\mu_{\widetilde{F} \circ \widetilde{G}(\varepsilon)}(x) & =\bigwedge_{x=a b}\left(\mu_{\widetilde{F}(\varepsilon)}(a) \wedge \mu_{\widetilde{G}(\varepsilon)}(b)\right) \\
& \leq \bigwedge_{x y=a b}\left(\mu_{\widetilde{F}(\varepsilon)}(a) \wedge \mu_{\widetilde{G}(\varepsilon)}(b y)\right) \\
& \leq \bigwedge_{x y=c d}\left(\mu_{\widetilde{F}(\varepsilon)}(c) \wedge \mu_{\widetilde{G}(\varepsilon)}(d)\right) \\
& =\mu_{\widetilde{F} \circ \widetilde{G}(\varepsilon)}(x y) .
\end{aligned}
$$

Similarly, we have $\mu_{\widetilde{F} \circ \widetilde{G}(\varepsilon)}(x y) \geq \mu_{\widetilde{F} \circ \widetilde{G}(\varepsilon)}(y)$, and so

$$
\mu_{\widetilde{F} \circ \widetilde{G}(\varepsilon)}(x y) \geq \mu_{\widetilde{F} \circ \widetilde{G}(\varepsilon)}(x) \wedge \mu_{\widetilde{F} \circ \widetilde{G}(\varepsilon)}(y) .
$$

In a similar way, we prove that

$$
v_{\widetilde{F} \circ \widetilde{G}(\varepsilon)}(x y) \leq v_{\widetilde{F} \circ \widetilde{G}(\varepsilon)}(x) \vee v_{\widetilde{F} \circ \widetilde{G}(\varepsilon)}(y),
$$

and the rest of the other cases can be similarly proved. Therefore, $(\widetilde{F}, A) \circ(\widetilde{G}, B)$ is an intuitionistic fuzzy soft ring over $R$.
Definition 33. Let $I$ be an ideal of $R$ and let $(\widetilde{F}, I)$ be an intuitionistic fuzzy soft set over $R$. Then, $(\widetilde{F}, I)$ is an intuitionistic fuzzy soft ideal of $R$, which will be denoted by $(\widetilde{F}, I) \widetilde{D} R$, if for each $x, y \in I$ and $r \in R$ the following conditions hold:

(i) $\mu_{\widetilde{F}(\varepsilon)}(x-y) \geq \mu_{\widetilde{F}(\varepsilon)}(x) \wedge \mu_{\widetilde{F}(\varepsilon)}(y)$ and $v_{\widetilde{F}(\varepsilon)}(x-y) \leq$ $v_{\widetilde{F}(\varepsilon)}(x) \vee v_{\widetilde{F}(\varepsilon)}(y)$.

Theorem 34. One has

(ii) $\mu_{\widetilde{F}(\varepsilon)}(x r) \geq \mu_{\widetilde{F}(\varepsilon)}(x)$ and $v_{\widetilde{F}(\varepsilon)}(x r) \leq v_{\widetilde{F}(\varepsilon)}(x)$,

(iii) $\mu_{\widetilde{F}(\varepsilon)}(r x) \geq \mu_{\widetilde{F}(\varepsilon)}(x)$ and $v_{\widetilde{F}(\varepsilon)}(r x) \leq v_{\widetilde{F}(\varepsilon)}(x)$.

Theorem 35. For any intuitionistic fuzzy soft ideals $(\widetilde{F}, I)$ and $(\widetilde{G}, J)$ over $R$, where $I \cap J \neq \emptyset$, one has $(\widetilde{F}, I) \widetilde{\cap}(\widetilde{G}, J) \widetilde{\triangleright} R$.

Proof. Since $I, J \triangleright R$, then $I \cap J \triangleright R$. By Definition 2.5, we can write $(\widetilde{F}, I) \widetilde{\cap}(\widetilde{G}, J)=(\widetilde{H}, S)$, where $S=I \cap J$, and for all $\varepsilon \in S=I \cap J, \mu_{\widetilde{H}(\varepsilon)}=\mu_{\widetilde{F}(\varepsilon)} \cap \mu_{\widetilde{G}(\varepsilon)}$. Then, for all $x, y \in I \cap J$ and $r \in R$,

$$
\begin{aligned}
\mu_{\widetilde{H}(\varepsilon)}(x-y)= & \mu_{\widetilde{F}(\varepsilon)}(x-y) \cap \mu_{\widetilde{G}(\varepsilon)}(x-y) \\
\geq & \left(\mu_{\widetilde{F}(\varepsilon)}(x) \wedge \mu_{\widetilde{F}(\varepsilon)}(y)\right) \\
& \cap\left(\mu_{\widetilde{G}(\varepsilon)}(x) \wedge \mu_{\widetilde{G}(\varepsilon)}(y)\right) \\
= & \left(\mu_{\widetilde{F}(\varepsilon)}(x) \wedge \mu_{\widetilde{G}(\varepsilon)}(x)\right) \\
& \wedge\left(\mu_{\widetilde{F}(\varepsilon)}(y) \wedge \mu_{\widetilde{G}(\varepsilon)}(y)\right) .
\end{aligned}
$$

Since $(\widetilde{F}, I) \widetilde{\triangleright} R$ and $(\widetilde{G}, J) \widetilde{\triangleright} R$, we know that $\mu_{\widetilde{F}(\varepsilon)}$ and $\mu_{\widetilde{G}(\varepsilon)}$ are intuitionistic fuzzy soft ideals of $R$. For all $\varepsilon \in S$, we have

$$
\begin{aligned}
\mu_{\widetilde{H}(\varepsilon)}(x-y) & \geq \mu_{\widetilde{H}(\varepsilon)}(x) \wedge \mu_{\widetilde{H}(\varepsilon)}(y), \\
\mu_{\widetilde{H}(\varepsilon)}(x r) & =\mu_{\widetilde{F}(\varepsilon)}(x r) \cap \mu_{\widetilde{G}(\varepsilon)}(x r) \\
& \geq \mu_{\widetilde{F}(\varepsilon)}(x) \cap \mu_{\widetilde{G}(\varepsilon)}(x) \\
& =\mu_{\widetilde{H}(\varepsilon)}(x) .
\end{aligned}
$$

We obtain a similar result for $v_{\widetilde{F}(\varepsilon)}$. Hence, $(\widetilde{H}, S)$ is an intuitionistic fuzzy soft ideal. Therefore, $(\widetilde{F}, I) \widetilde{\cap}(\widetilde{G}, J)=(\widetilde{H}, S) \widetilde{\triangleright} R$.

Theorem 36. For any fuzzy soft ideals $(f, I)$ and $(g, J)$ over $R$, in which $I$ and $J$ are disjoint, one has $(f, I) \widetilde{\cup}(g, J) \widetilde{\triangleright} R$.

Proof. Assume that $(f, I) \widetilde{\triangleright} R$ and $(g, J) \widetilde{\triangleright R}$. By means of definition we can write $(f, I) \widetilde{\cup}(g, J)=(h, K)$, where $K=I \cup J$ and for every $\mu \in K$

$$
h_{\mu}(c)= \begin{cases}f_{\mu} & \text { if } c \in I-J \\ g_{\mu} & \text { if } c \in J-I \\ f_{\mu} \vee g_{\mu} & \text { if } c \in I \cap J .\end{cases}
$$

Since $I \cap J=\emptyset$, either $c \in I-J$ or $c \in J-I$ for all $c \in K$. If $c \in$ $I-J$, the $h_{\mu}=f_{\mu}$ is an ideal of $R \operatorname{since}(f, I) \widetilde{\triangleright} R$. If $c \in J-I$, 
then $h_{\mu}=g_{\mu}$ is an ideal of $R$ since $(g, J) \widetilde{\triangleright} R$. Thus, $h_{\mu}$ is an ideal of $R$ for all $c \in K$, and so $(f, I) \widetilde{\cup}(g, J)=(h, K) \widetilde{\triangleright R}$.

Theorem 37. Let $(\widetilde{F}, I)$ be an intuitionistic fuzzy soft ideal over R. If $\left\{\left(\widetilde{F}_{j}, I_{j}\right) \mid j \in J\right\}$ is a nonempty family of intuitionistic fuzzy soft ideals of $(\widetilde{F}, I)$, where $J$ is an index set, then,

(1) $\widetilde{\cap}_{j \in J}\left(\widetilde{F}_{j}, I_{j}\right) \widetilde{\triangleright R}$,

(2) $\widetilde{\wedge}_{j \in J}\left(\widetilde{F}_{j}, I_{j}\right) \widetilde{\triangleright} R$,

(3) $\widetilde{\nabla}_{j \in J}\left(\widetilde{F}_{j}, I_{j}\right) \widetilde{\triangleright} R$, where $I_{j} \cap I_{k}=\{0\}$ for all $j, k \in J$.

Definition 38. Let $f: R \rightarrow S$ and $g: A \rightarrow B$ be two functions, where $A$ and $B$ are parameter sets for intuitionistic fuzzy soft sets $R$ and $S$, respectively. Then, the pair $(f, g)$ is called an intuitionistic fuzzy soft function from $R$ to $S$.

Definition 39. Let $(\widetilde{F}, A)$ and $(\widetilde{G}, B)$ be two intuitionistic fuzzy soft rings over $R$ and $S$, respectively, let $f: R \rightarrow S$ be a homomorphism of rings, and let $g: A \rightarrow B$ be a mapping of sets. Then, we say that $(f, g):(\widetilde{F}, A) \rightarrow(\widetilde{G}, B)$ is an intuitionistic fuzzy soft homomorphism of intuitionistic fuzzy soft rings and define by $f(\widetilde{F}, A)=(\widetilde{G}, B) g$, if the following conditions are satisfied:

$$
\begin{aligned}
& f\left(\mu_{\widetilde{F}(\varepsilon)}(x)\right)=\left(\mu_{\widetilde{G}(\varepsilon)}(x)\right) g, \\
& f\left(v_{\widetilde{F}(\varepsilon)}(x)\right)=\left(v_{\widetilde{G}(\varepsilon)}(x)\right) g .
\end{aligned}
$$

Definition 40. If there exists an intuitionistic fuzzy soft ring homomorphism between $(\widetilde{F}, A)$ and $(\widetilde{G}, B)$, we say that $(\widetilde{F}, A)$ is intuitionistic fuzzy soft homomorphic to $(\widetilde{G}, B)$ and is denoted by $(\widetilde{F}, A) \sim(\widetilde{G}, B)$. Moreover, if $f$ is an isomorphism, then $(\widetilde{F}, A)$ is intuitionistic fuzzy soft isomorphic to $(\widetilde{G}, B)$, which is denoted by $(\widetilde{F}, A) \simeq(\widetilde{G}, B)$.

Now, we show that the homomorphic image and preimage of an intuitionistic fuzzy soft ring are also intuitionistic fuzzy soft rings.

Definition 41. Let $(\widetilde{F}, A)$ and $(\widetilde{G}, B)$ be two intuitionistic fuzzy soft rings over $R$ and $S$. Let $(f, g)$ be an intuitionistic fuzzy soft function from $R$ to $S$.

(1) The image of $(\widetilde{F}, A)$ under the intuitionistic fuzzy soft function $(f, g)$ denoted by $(f, g)(\widetilde{F}, A)$ is the intuitionistic fuzzy soft ring over $S$ defined by $(f, g)$ $(\widetilde{F}, A)=(f(\widetilde{F}), g(A))$, where

$$
\begin{aligned}
& f(\widetilde{F})_{k}(s)= \begin{cases}\bigvee_{f(r)=s g(a)=k} \bigvee_{\widetilde{F}(\varepsilon)}(r) & \text { if } r \in f^{-1}(s) \\
0 & \text { otherwise, }\end{cases} \\
& f(\widetilde{F})_{k}(s)= \begin{cases}\bigwedge_{f(r)=s g(a)=k} \bigwedge_{\widetilde{F}(\varepsilon)}(x) & \text { if } r \in f^{-1}(s) \\
1 & \text { otherwise, }\end{cases}
\end{aligned}
$$

for all $k \in g(A)$ and for all $s \in S$.
(2) The preimage of $(\widetilde{G}, B)$ under the intuitionistic fuzzy soft function $(f, g)$ denoted by $(f, g)^{-1}(\widetilde{G}, B)$ is the intuitionistic fuzzy soft ring over $R$ defined by $(f, g)^{-1}$ $(\widetilde{G}, B)=\left(f^{-1}(\widetilde{G}), g^{-1}(B)\right)$, where $f^{-1}(\widetilde{G})_{a}(r)=$ $(\widetilde{G})_{g(a)}(f(r))$, for all $a \in g^{-1}(B)$ and for all $r \in R$.

If $f$ and $g$ are injective (surjective), then $(f, g)$ is said to be injective (surjective).

Definition 42. Let $(f, g)$ be an intuitionistic fuzzy soft function from $R$ to $S$. If $f$ is a homomorphism function from $R$ to $S$, then $(f, g)$ is said to be intuitionistic fuzzy soft homomorphism. If $f$ is an isomorphism from $R$ to $S$ and $g$ is a one-to-one mapping from $A$ onto $B$, then $(f, g)$ is said to be an intuitionistic fuzzy soft isomorphism.

Theorem 43. Let $(\widetilde{F}, A)$ be an intuitionistic fuzzy soft ring over $R$ and $(f, g)$ an intuitionistic fuzzy soft homomorphism from $R$ to $S$. Then, $(f, g)(\widetilde{F}, A)$ is an intuitionistic fuzzy soft ring over $S$.

Proof. The proof is straightforward.

Theorem 44. Let $(\widetilde{G}, B)$ be an intuitionistic fuzzy soft ring over $S$ and $(f, g)$ be an intuitionistic fuzzy soft homomorphism from $R$ to $S$. Then, $(f, g)^{-1}(\widetilde{G}, B)$ is an intuitionistic fuzzy soft ring over $R$.

Proof. The proof is straightforward.

\section{Conclusion}

In this work the theoretical point of view of intuitionistic fuzzy soft sets in ring and ideal is discussed. The work is focused on intuitionistic fuzzy soft rings and fuzzy soft ideals. These concepts are basic structures for improvement of fuzzy soft set theory. One can extend this work by studying other algebraic structures.

\section{Acknowledgment}

The authors are highly grateful to the referees for their valuable comments and suggestions for improving the paper.

\section{References}

[1] D. Molodtsov, "Soft set theory-first results," Computers \& Mathematics with Applications, vol. 37, no. 4-5, pp. 19-31, 1999.

[2] P. K. Maji, R. Biswas, and A. R. Roy, "Soft set theory," Computers \& Mathematics with Applications, vol. 45, no. 4-5, pp. 555-562, 2003.

[3] A. R. Roy and P. K. Maji, "A fuzzy soft set theoretic approach to decision making problems," Journal of Computational and Applied Mathematics, vol. 203, no. 2, pp. 412-418, 2007.

[4] B. X. Yao, J. L. Liu, and R. X. Yan, "Fuzzy soft set and soft fuzzy set," in Proceedings of the 4th International Conference on Natural Computation (ICNC '08), pp. 252-255, Jinan, China, October 2008 . 
[5] K. T. Atanassov, Intuitionistic Fuzzy Sets, Theory and Applications, vol. 35 of Studies in Fuzziness and Soft Computing, Physica, Heidelberg, Germany, 1999.

[6] K. T. Atanassov, "Remarks on the intuitionistic fuzzy sets. III," Fuzzy Sets and Systems, vol. 75, no. 3, pp. 401-402, 1995.

[7] S. K. De, R. Biswas, and A. R. Roy, "Some operations on intuitionistic fuzzy sets," Fuzzy Sets and Systems, vol. 114, no. 3, pp. 477-484, 2000.

[8] B. Davvaz, P. Corsini, and V. Leoreanu-Fotea, "Atanassov's intuitionistic $(S, T)$-fuzzy $n$-ary sub-hypergroups and their properties," Information Sciences, vol. 179, no. 5, pp. 654-666, 2009.

[9] B. Davvaz and W. A. Dudek, "Intuitionistic fuzzy $H_{v}$-ideals," International Journal of Mathematics and Mathematical Sciences, vol. 2006, Article ID 65921, 11 pages, 2006.

[10] B. Davvaz, W. A. Dudek, and Y. B. Jun, "Intuitionistic fuzzy $H_{v^{-}}$ submodules," Information Sciences, vol. 176, no. 3, pp. 285-300, 2006.

[11] B. Davvaz and S. K. Majumder, "Atanassov's intuitionistic fuzzy interior ideals of G-semigroups," UPB Scientific Bulletin, Series A, vol. 73, no. 3, pp. 45-60, 2011.

[12] W. A. Dudek, B. Davvaz, and Y. B. Jun, "On intuitionistic fuzzy sub-hyperquasigroups of hyperquasigroups," Information Sciences, vol. 170, no. 2-4, pp. 251-262, 2005.

[13] L. A. Zadeh, "Fuzzy sets," Information and Computation, vol. 8, pp. 338-353, 1965.

[14] P. K. Maji, R. Biswas, and A. R. Roy, "Fuzzy soft sets," Journal of Fuzzy Mathematics, vol. 9, no. 3, pp. 589-602, 2001.

[15] P. K. Maji, R. Biswas, and A. R. Roy, "Intuitionistic fuzzy soft sets," Journal of Fuzzy Mathematics, vol. 9, no. 3, pp. 677-692, 2001.

[16] P. K. Maji, A. R. Roy, and R. Biswas, "Intuitionistic fuzzy soft sets," Journal of Fuzzy Mathematics, vol. 9, no. 3, pp. 677-692, 2001.

[17] E. İnan and M. A. Öztürk, "Fuzzy soft rings and fuzzy soft ideals," Neural Computing and Applications, vol. 21, no. 1, supplement, pp. 1-8, 2011. 


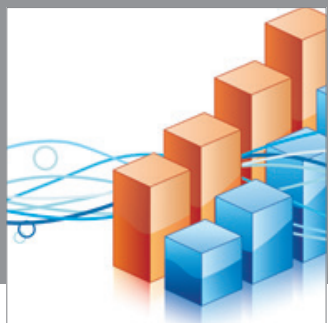

Advances in

Operations Research

mansans

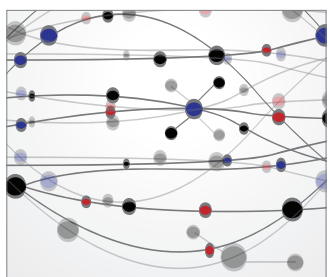

The Scientific World Journal
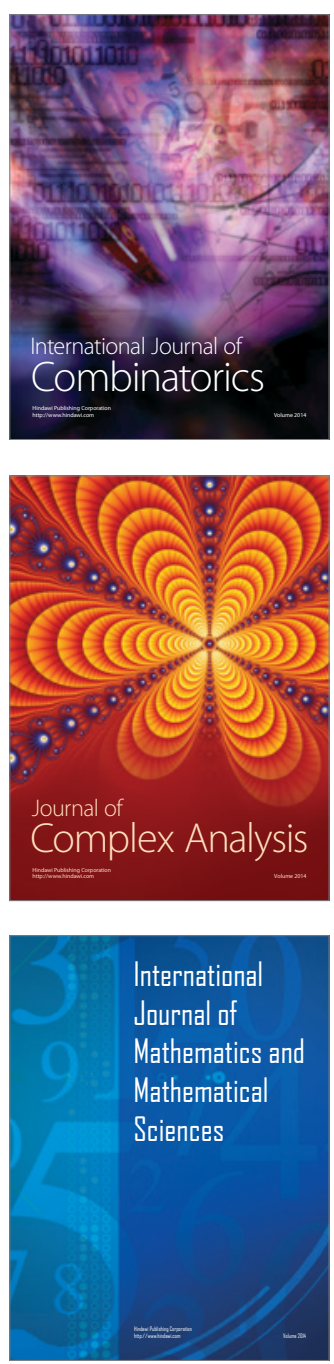
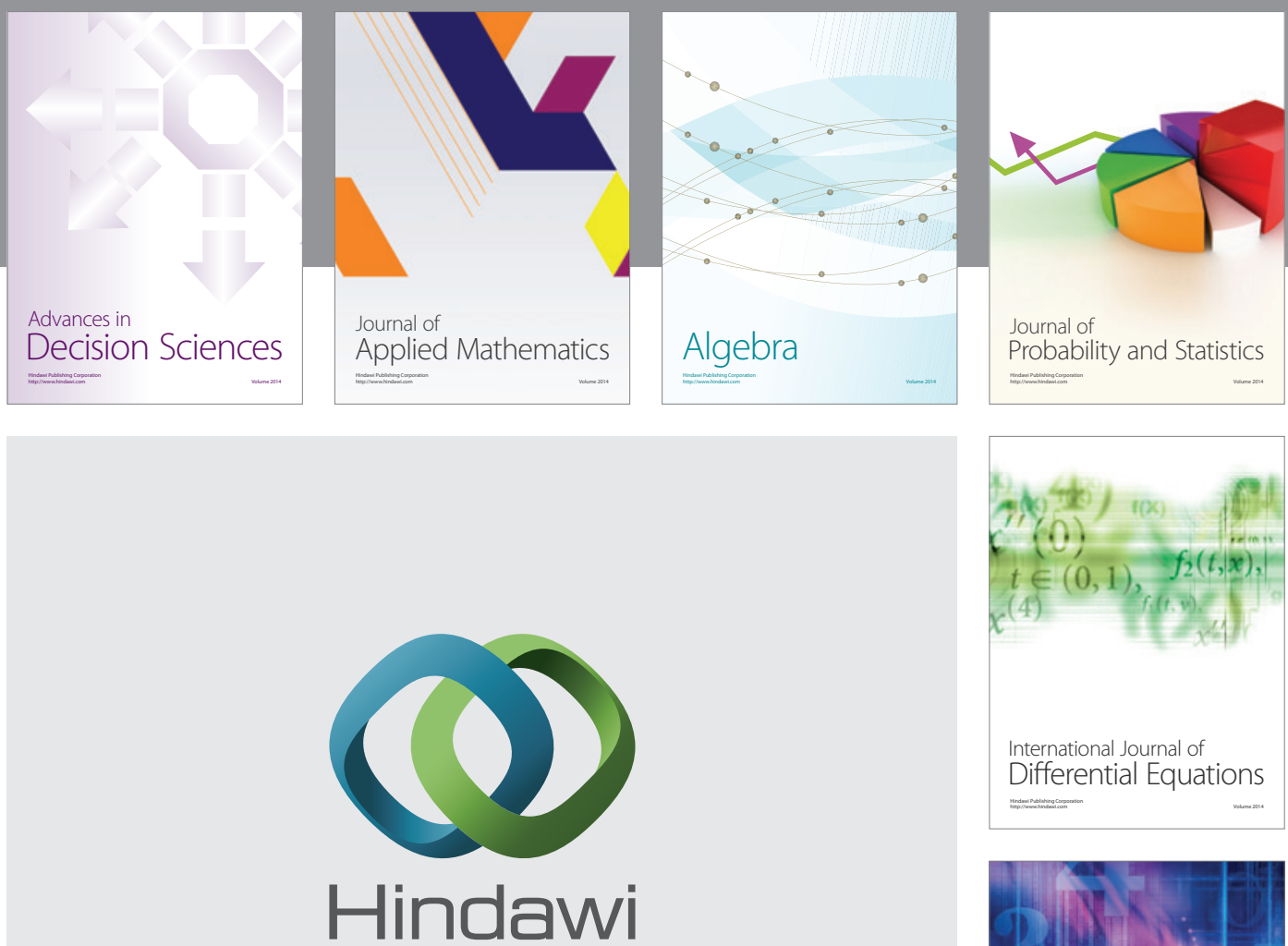

Submit your manuscripts at http://www.hindawi.com
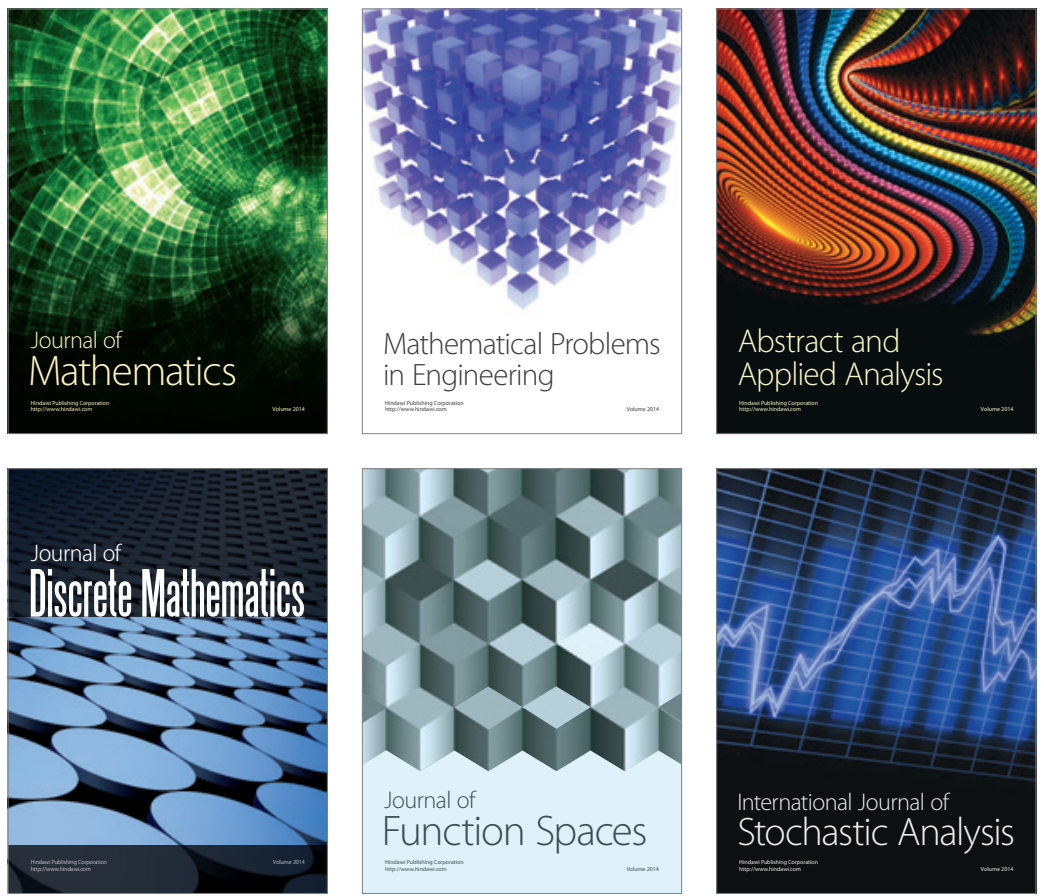

Journal of

Function Spaces

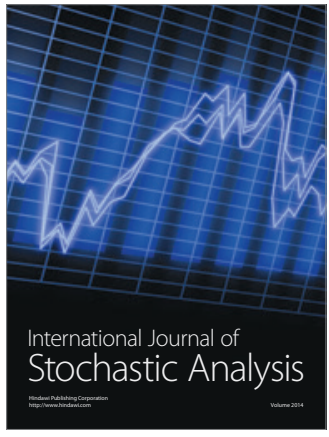

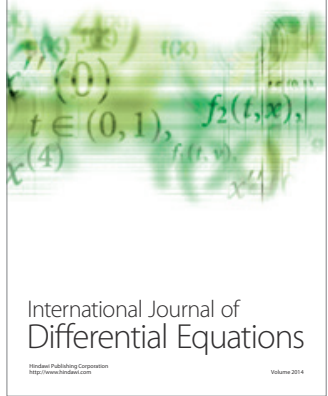
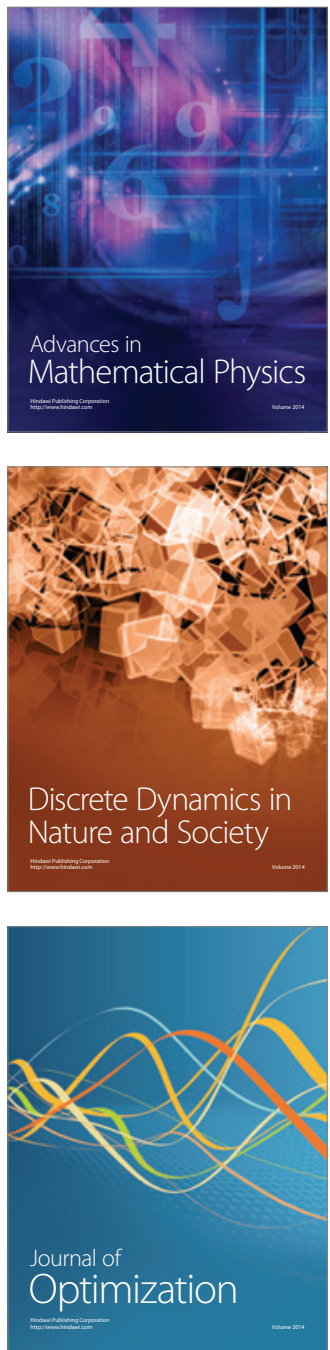